\title{
HUMAN PLACENTAL LIPID PEROXIDATION-II. NADPH AND IRON DEPENDENT STIMULATION OF MICROSOMAL LIPID PEROXIDATION BY PARAQUAT
}

\author{
Michael F. Kenel, ${ }^{*}$ Lorelle L. Bestervelt and Arun P. Kulkarni \\ Toxicology Program, Department of Environmental and Industrial Health, School of Public Health, \\ The University of Michigan, Ann Arbor, MI 48109-2029, U.S.A.
}

(Received 15 September 1986)

\begin{abstract}
Paraquat, a widely used herbicide, was found to cause a marked stimulation of lipid peroxidation in the human placental microsomes in vitro.

2. Both NADPH and chelated iron were necessary to observe paraquat-stimulated lipid peroxidation.

3. The malondialdehyde accumulation in the incubation medium increased with increase in time, protein and paraquat concentration.

4. The reaction did not exhibit the initial lag phase suggesting that endogenous membrane-bound antioxidants in human placental microsomes are either absent or present in extremely small quantities.
\end{abstract}

\section{INTRODUCTION}

Tissue lipid peroxidation is a degradative free-radical process that impairs the structural integrity and function of biomembranes (Plaa and Witschi, 1976; Bus and Gibson, 1979; Slater, 1984; Aust, et al., 1985). It has been implicated as a major biochemical mechanism of tissue damage associated with the exposure to certain environmental chemicals and drugs (Plaa and Witschi, 1976; Bus and Gibson, 1979; Slatter, 1984; Aust et al., 1985). Stimulation of human placental microsomal lipid peroxidation by NADPH and iron has been previously studied in our laboratory (Kenel and Kulkarni, 1982; Kulkarni and Kenel, 1987) as well as by other investigators (Diamant et al., 1980).

Exposure during pregnancy to environmental chemicals capable of stimulating lipid peroxidation within placental membranes is of toxicological significance since it may alter the structure and function of the placenta in such a way that the foetus is at an increased risk. Paraquat, a widely used herbicide, is a well-known pulmonary toxicant (Smith and Heath, 1976; Haley, 1979). The reports of animal studies designed to evaluate reproductive hazards of paraquat have documented its transplacental transfer, increased embryo/fetal mortality, reduced growth rate, and high mutagenic potential (Bus et al., 1975; Hoffman and Eastin, 1982; Selypes et al., 1982; Ingebrigtsen et al., 1985). Although very little is known about reproductive toxicity of paraquat in humans, at least two separate incidences of paraquat poisoning of pregnant women have been reported in the literature: one involving an accidental exposure

*Present address: Drug Safety Evaluations, McNeil Pharmaceutical, Spring House, PA 19477, U.S.A $\dagger$ To whom reprint requests should be addressed.
(Finnelly et al., 1968) and the other was a suicide attempt (Musson and Porter, 1982).

Bus et al. (1974) initially proposed stimulation of microsomal lipid peroxidation as the underlying biochemical mechanism of paraquat lung toxicity. It was suggested that single electron reduction of paraquat, mediated by NADPH cytochrome-C reductase, results in a reduced radical species capable of rapidly reacting with molecular oxygen to produce superoxide anion. The cyclic reduction-oxidation which continuously generates reactive oxygen species is believed to trigger peroxidation of membrane lipids (Bus and Gibson, 1979; Aust et al., 1985). However, the available reports on this subject are not consistent. Thus a species- and/or tissue-specific stimulation (Shu et al., 1979; Talcott et al., 1979; Trush et al., 1981; Kulkarni and Hodgson, 1981) or inhibition (Ilett et al., 1974; Steffen and Netter, 1980; Kornbrust and Mavis, 1980; Misra and Gorsky, 1981; Montgomery and Niewoehner, 1979) of microsomal lipid peroxidation by paraquat was observed by different investigators. According to Trush et al. (1981) many of the negative findings may have been related to the presence of high levels of tissue antioxidants and/or suboptimal assay conditions.

Since there exists some controversy in the literature concerning paraquat and tissue lipid peroxidation, the present work was undertaken to study the effects of paraquat on the NADPH-dependent lipid peroxidation in human placental microsomes. The results reported in this paper confirm our earlier observations (Kenel and Kulkarni, 1982; Kulkarni and Kenel, 1987) and those of Diamant et al. (1980) on the occurrence of NADPH-supported lipid peroxidaton in microsomes of human term placenta Furthermore, the evidence presented indicates that paraquat has stimulatory effects on the human placental microsomal lipid peroxidation. 


\section{MATERIALS AND METHODS}

Chemicals

NADPH, Tris- $\mathrm{HCl}$, disodium salt of ethyleneglycol-bis( $\beta$-aminoethylether)- $N, N, N^{\prime}, N^{\prime}$-tetraacetic acid (EGTA), disodium salt of ethylenediaminetetraacetic acid (EDTA), sucrose, and thiobarbituric acid (TBA) were purchased from the Sigma Chemical Co., St Louis, Mo. Reagent grade $\mathrm{CCl}_{4}$ was obtained from Fisher Chemical Co.; Paraquat (methyl viologen) was a product of Aldrich Chemical $\mathrm{Co}$.

\section{Isolation of microsomes}

Full term placentas from healthy women were immediately placed on ice and processed within an hour of delivery. Microsomes were isolated by differential centrifugation as described previously (Kulkarni et al., 1985). Unless otherwise stated, placental homogenates $(20 \%,(w / v)$ were prepared in a medium containing $0.02 \mathrm{M}$ Tris $\mathrm{HCl}$ buffer, $\mathrm{pH}$ 7.4, 0.25 $\mathrm{M}$ sucrose, and $0.5 \mathrm{mM}$ disodium EGTA (Diamant et al, 1980). In few experiments (as specified in the Results Section) isolation medium contained EDTA in place of EGTA. Microsomes stored $\left(0-15\right.$ days at $\left.-20^{\circ} \mathrm{C}\right)$ in homogenization medium were diluted several fold with

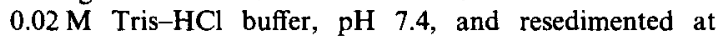
$105,000 \mathrm{~g}$ for $60 \mathrm{~min}$ to remove the chelating agent and to minimize cytosolic contaminants. The washed microsomes were suspended in $0.02 \mathrm{M}$ Tris- $\mathrm{HCl}$ buffer, $\mathrm{pH} 7.4$. The rate of lipid peroxidation did not change significantly $(<10 \%)$ during storage.

\section{Paraquat-stimulated lipid peroxidation}

Based on preliminary experiments, the optimal assay conditions (except as noted otherwise) were found to be incubation at $37^{\circ} \mathrm{C}$ of reaction mixtures $(1.0 \mathrm{ml}$ final volume) containing: $0.2 \mathrm{mg}$ microsomal protein $/ \mathrm{ml}, 0.02 \mathrm{M}$ Tris- $\mathrm{HCl}$ buffer, $\mathrm{pH} 7.4$ and indicated concentrations of paraquat and NADPH. Microsomes were pre-incubated in the presence of paraquat for $5 \mathrm{~min}$ at $37^{\circ} \mathrm{C}$ before starting the reaction with NADPH. In some experiments, desired concentration of chelated iron (premixed EDTA and $\mathrm{FeCl}_{2}$, in $2: 3$ ratio) was added to the incubation media in addition to or in the absence of paraquat. The incubation media containing no NADPH served as controls. The termination of reaction and the estimation of the amount of malondialdehyde (MDA) as the TBA-reactive material were carried out as described previously (Kulkarni and Hodgson, 1981; Kulkarni and Kenel, 1987). To prevent non-specific chromophore formation during boiling, butylated hydroxytoluene (BHT) $(0.01 \%)$ was added (Kulkarni and Hodgson, 1981).

Protein content was determined by the Biuret method using bovine serum albumin as a standard (Gornall et al., 1949).

\section{RESULTS}

Lipid peroxidation was stimulated by paraquat when human placental microsomes were incubated aerobically in the presence of NADPH. The timedependent increase in the accumulation of TBAreactive material in the reaction medium in the presence or absence of paraquat is shown in Fig. 1. Both iron- as well as paraquat-stimulated lipid peroxidation reaction was linear up to approximately 5 and $10 \mathrm{~min}$ respectively. In the absence of exogenous iron, an initial rate of $2.23 \pm 0.03 \mathrm{nmol}$ of MDA production $/ \mathrm{min} / \mathrm{mg}$ protein was observed in the presence of $2 \mathrm{mM}$ paraquat (Fig. 1). This rate is about $50-70 \%$ of the rate observed in a chelated iron dependent reaction. $\mathrm{CCl}_{4}$, which is known to stimulate lipid peroxidation in the hepatic microsomes

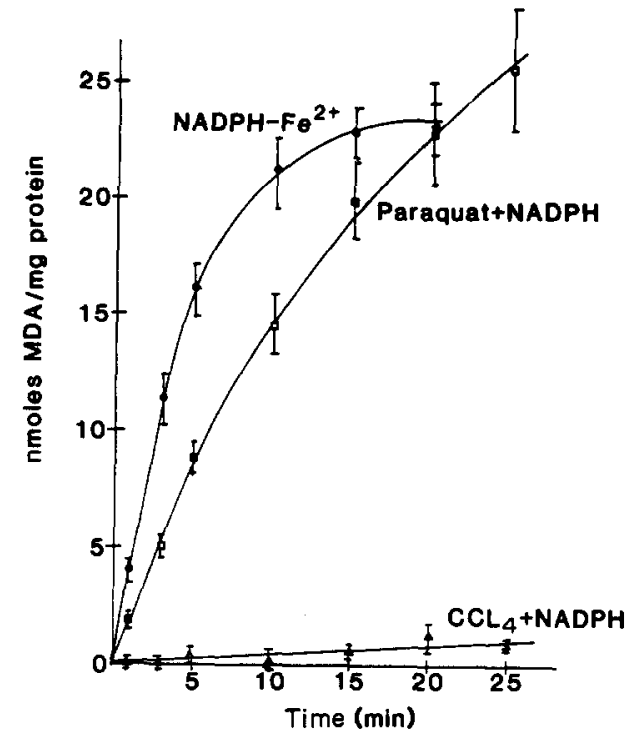

Fig. 1. Time dependence of human placental microsomal lipid peroxidation. Placental tissue was homogenized in the medium containing EGTA. Washed microsomes were incubated in Tris buffer for the indicated time period in the presence of $0.2 \mathrm{mM}$ NADPH plus chelated iron (-C), or $2.0 \mathrm{mM}$ paraquat plus $0.4 \mathrm{mM}$ NADPH $(\square-\square)$ or $1.0 \mu 1$ of $\mathrm{CCl}_{4} / \mathrm{ml}$ plus $0.4 \mathrm{mM}$ NADPH $(\Delta-\Delta)$ as described under Materials and Methods. Each point represents the mean $\pm \operatorname{SE}(n=4)$ after subtraction of the amount of MDA formed in the respective control incubation in the absence of NADPH.

(Kulkarni and Hodgson, 1981) was also tested. In contrast to the results obtained in the presence of paraquat, $\mathrm{CCl}_{4}$ did not cause marked stimulation of lipid peroxidation, due probably to lack of its metabolism to $\mathrm{CCl}_{3}$ by the placental microsomes

Similar to the $\mathrm{Fe}^{2+}-\mathrm{NADPH}$ system (Kulkarni and Kenel, 1987), the specific activity of the paraquat stimulated lipid peroxidation was maximum when protein concentration of $0.2 \mathrm{mg} / \mathrm{ml}$ (Fig. $2 \mathrm{~A}$ ) was used. The reasons for the observed decrease in the specific activity when higher $(>0.2 \mathrm{mg} / \mathrm{ml})$ protein concentrations were used is not clear at present. Paraquat stimulated MDA production required NADPH and $0.4 \mathrm{mM}$ NADPH appeared to be adequate (Fig. 2B) to support the lipid peroxidation process maximally under the experimental conditions employed. Lipid peroxidation rates increased with an increase in paraquat concentration (Fig. 2C). The paraquat concentration causing half the maximal stimulation of lipid peroxidation was found to be about $0.75 \mathrm{mM}$ and the enhancement was detectable at the lowest concentration of paraquat $(0.1 \mathrm{mM})$ tested.

The addition of chelated iron to the paraquat and NADPH supplemented reaction media produced variable results and either no effect or a $20-66 \%$ further stimulation in MDA production was observed in some experiments. The paraquat-stimulated lipid peroxidation in the presence or absence of exogenous iron was completely abolished when excess EDTA $(0.1-1.0 \mathrm{mM})$ was incorporated into the incubation media. These results suggested that iron 


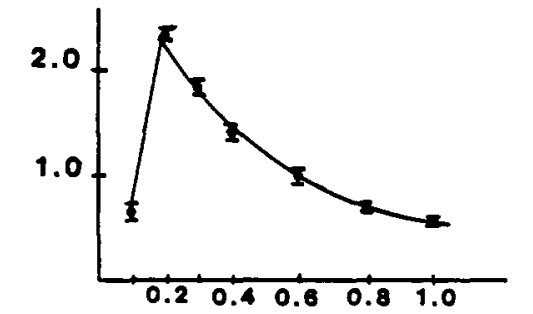

A
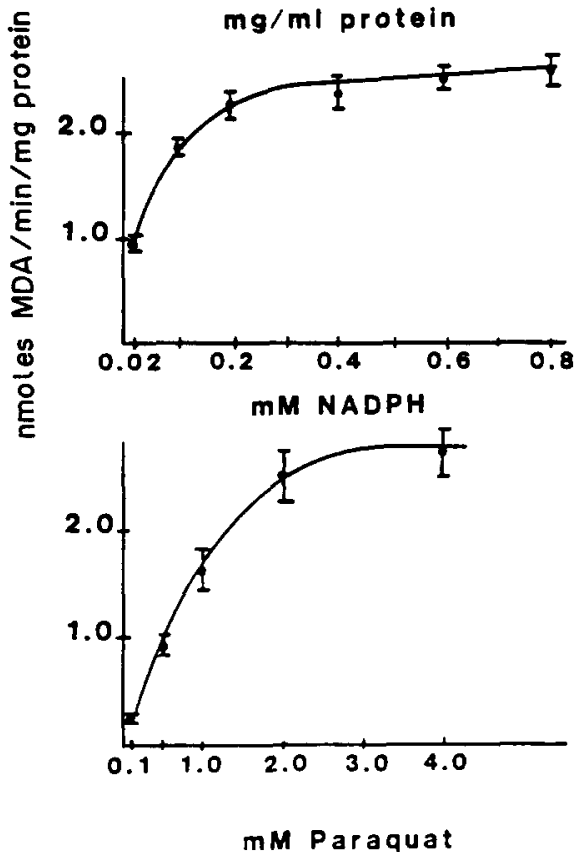

Fig. 2. The effect of protein (A), NADPH (B), and paraquat (C) concentration on lipid peroxidation of human placental microsomes. Placental tissue was homogenized in the medium containing EGTA. Washed microsomes were incubated in Tris buffer for $5.0 \mathrm{~min}$ in the presence of paraquat, and NADPH as described under Materials and Methods. Each point represents the mean $\pm S E(n=4)$ after subtraction of the amount of MDA formed in the respective control incubation in the absence of NADPH.

or other reduced transitional metal is required to observed paraquat stimulated lipid peroxidation in human placental microsomes. Therefore, the observed paraquat-stimulated lipid peroxidation in microsomes isolated using medium containing EGTA (Fig. 1) may be due to trace metal contamination.

To further establish the iron-dependency of paraquat-stimulated lipid peroxidation, additional experiments were performed using washed microsomes isolated from placental homogenates prepared in a medium containing EDTA (in place of EGTA). Without chelated iron, no microsomal lipid peroxidation was observed in the presence of paraquat and NADPH. The results (Fig. 3) indicated that paraquat-stimulation of lipid peroxidation depends upon the amount of chelated iron present in the incubation medium. A significant stimulation (3-4-fold) was noted at the lowest concentration $(0.1 \mu \mathrm{M})$ of chelated iron tested and the stimulation was maximum (about 10-15-fold in different experi- ments) when $10 \mu \mathrm{M}$ chelated iron was present. Further increase in the concentrations of chelated iron did not yield a proportional enhancement of lipid peroxidation.

\section{DISCUSSION}

The results obtained in this study confirm our earlier findings (Kenel and Kulkarni, 1982; Kulkarni and Kenel, 1987) and those of Diamant et al. (1980) on the occurrence of a NADPH-supported lipid peroxidation process in the microsomes of human term placenta.

In recent years, the stimulated membrane lipid peroxidation as a mechanism of organ toxicity of paraquat has become a subject of intense debate. Thus, the reported stimulation (Bus et al., 1974; Shu et al., 1979; Talcott et al., 1979; Trush et al., 1981; Kulkarni and Hodgson, 1981) and inhibition (Ilett $e t$ al., 1974; Montgomery and Niewoehner, 1979; Steffen and Netter, 1980; Kornbrust and Mavis, 1980; Misra and Gorsky, 1981; Klimel et al., 1982) of lipid peroxidation indicate that both species and tissue-specific biochemical differences may exist that determine the in vivo and in vitro response. Previously Kornbrust and Mavis (1980) reported that paraquat did not stimulate lipid peroxidation when added to human lung microsomes in the presence of NADPH. To our knowledge, similar studies have not been performed with other human tissues including placenta. It should be pointed out that suboptimal assay conditions were reported to be at least partly responsible for the reported negative in vitro findings (Trush et al., 1981). Considering these reports and the facts that paraquat concentrates in the guinea pig and rat placenta (Ingebrigtsen et al., 1985) and many animal studies (Bus et al., 1975; Hoffman and Eastin, 1982; Selypes et al., 1982; Ingebrigtsen et al., 1985)

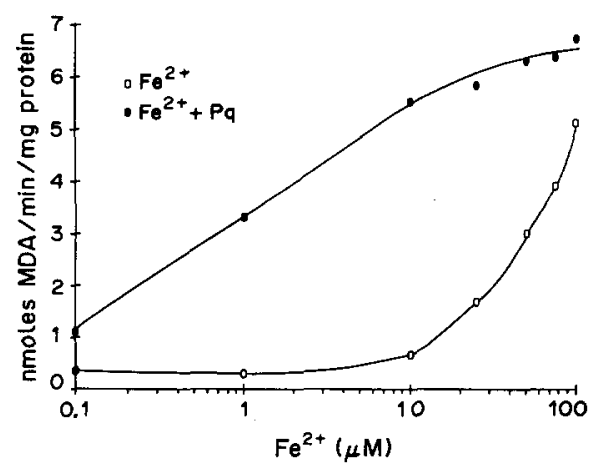

Fig. 3. Dependency of paraquat-stimulated lipid peroxidation of human placental microsomes on chelated iron. Placental tissue was homogenized in the medium containing EDTA. Washed microsomes were incubated in Tris buffer for $5.0 \mathrm{~min}$ in the presence of $0.4 \mathrm{mM} \mathrm{NADPH}$ and the indicated concentrations of chelated iron $\left(\mathrm{O}_{--}-\mathrm{O}\right)$ or in the presence of $0.4 \mathrm{mM}$ NADPH, the indicated concentrations of chelated iron and $2.0 \mathrm{mM}$ paraquat (--O) as described under Materials and Methods. The results of a typical experiment are shown. Similar results were observed in two other experiments. Each value is after subtraction of the amount of MDA formed in the respective control incubation in the absence of NADPH. 
have documented fetotoxicity of paraquat, it was viewed important to examine the response of human placental microsomes to this chemical.

Redox cycling of paraquat as well as certain other chemicals and the concomitant generation of reactive oxygen species at the expense of NADPH has been proposed as the molecular mechanism of their cytotoxicity (Bus and Gibson, 1979). Formation of paraquat radical, in an enzymatic one-electron reduction, is the first required step followed by a reaction with molecular oxygen to yield the superoxide anion radical and subsequently, for example $\mathrm{H}_{2} \mathrm{O}_{2}, \mathrm{OH}^{\circ}$ and ${ }^{1} \mathrm{O}_{2}$. The attack of any of these reactive oxygen species and/or other initiating species on the polyunsaturated fatty acids present in membranes is associated with lipid peroxidation (Bus and Gibson, 1979; Aust et al., 1985). Talcott et al. (1979), on the other hand, proposed a mechanism of paraquatinitiated lipid peroxidation independent of superoxide and peroxide generation. Although the necessary evidence was not provided, the authors suggested that the ferrous ion initiates the reaction by reductive cleavage of preformed lipid hydroperoxide to alkoxyl radicals. However, an accumulation of large quantities of lipid hydroperoxides in biological membranes under normal physiological conditions is highly unlikely when various defense mechanisms are fully operational. Although the low blank values generally observed in our study support this contention, the presence of catalytic amounts of preformed lipid hydroperoxides in the preparations of placental microsomes used cannot be ruled out completely.

The data given in Figs 1-3 clearly suggest that paraquat is quite capable of stimulating lipid peroxidation in human placental microsomes. The paraquat-stimulated lipid peroxidation exhibited time- (Fig. 1) and protein-dependence and the requirement for NADPH (Fig. 2). The facts that paraquat stimulated lipid peroxidation was completely abolished by excess EDTA, and the reaction was markedly enhanced by small amount of chelated iron (Fig. 3) suggest an obligatory role for iron. This stimulatory response decreased with increasing iron concentration. Thus, not only the presence of iron is necessary, but its level determines the response. Failure to clearly recognize the critical role of iron concentration in the incubation medium on the paraquat-stimulated lipid peroxidation might have been yet another contributory factor responsible for the reported negative findings.

Recently, the ratio of $\mathrm{Fe}^{3+}: \mathrm{Fe}^{2+}$ was shown to be the primary determining factor for the initiation of lipid peroxidation (Braughler et al., 1986). Although further mechanistic studies are essential, it is tempting to speculate that the rate of reduction of $\mathrm{Fe}^{3+}$ to $\mathrm{Fe}^{2+}$ by the placental microsomal NADPH cytochrome $\mathrm{P}-450$ reductase is the rate limiting step in the NADPH-supported lipid peroxidation reaction triggered by chelated $\mathrm{Fe}^{2+}$. In the presence of paraquat, this reduction process occurs rapidly bringing the ratio in the optimal range to trigger intense lipid peroxidation. Our observations that the stimulatory effect of paraquat diminishes when increasing concentration of chelated $\mathrm{Fe}^{2+}$ is used (Fig. 3) is in accord with this proposal.
Although published data on placental content of lipid soluble antioxidants such as vitamin $\mathrm{E}$, are not available, a lack of initial lag (Fig. 1) in the lipid peroxidation process suggests their presence in extremely small quantities, if any. Furthermore, the reported low levels of reduced glutathione (Radulovic and Kulkarni, 1986), the water soluble antioxidant, in human placenta is expected to render cellular environment in vivo quite vulnerable to the attacks by reactive oxygen species and xenobiotic radicals leading to lipid peroxidation.

Similar to the liver, the presence of certain enzymatic defenses against oxygen radical toxicity such as superoxide dismutase (Sekiba and Yoshioka, 1979), catalase (Misra and Mukherjea, 1985), glutathione $S$-transferase (Radulovic and Kulkarni, 1985), glutathione peroxidase (Awasthi et al., 1979), and peroxidase (Matkovics, 1975; Nelson and Kulkarni, 1986) is known in human placenta. However, their effectiveness in regulating human placental lipid peroxidation is currently unknown.

Paradoxically, despite the presence and apparent function of different enzymatic and non-enzymatic defense mechanisms, it is accepted that certain xenobiotics can initiate membrane lipid peroxidation that leads to toxic lesions. If this is true, then the potential of placental damage via lipid peroxidation in humans exists following paraquat exposure during pregnancy. Although the biological consequences of lipid peroxidation of placental tissue are unknown at present, it is expected that it may trigger a chain of biochemical events leading to placental malfunction. In addition, the possibility that some highly cytotoxic products of lipid peroxidation such as 4-hydroxyalkenals may cross the placenta exposing directly the conceptus must be considered. Several lipid peroxidation products have been shown to inhibit key enzymes in intermediary metabolism, act as growth inhibitor, cytotoxic agent and act presumably as mutagenic and carcinogenic (Shamberger et al., 1974; Plaa and Witschi, 1976; Moody and Hassan, 1982; Benedetti et al., 1984).

Shu et al., (1979) reported that paraquat stimulated in vitro lipid peroxidation may not be related to its in vivo pulmonary toxicity. In view of this, it is essential to determine how much of a role lipid peroxidation contributes to the in vivo fetotoxicity of paraquat in mammals. Certainly, lipid peroxidation is not the only potentially destructive reaction mediated by paraquat and other xenobiotics capable of undergoing redox cycling. Nonetheless, the demonstration that paraquat can stimulate in vitro placental lipid peroxidation suggests that the potential does exist in vivo.

\section{SUMMARY}

Paraquat is an effective herbicide used extensively throughout the world. A number of animal reproductive toxicity studies reported in the literature have documented the fetotoxicity of paraquat. The reports on the accidental as well as deliberate exposure of pregnant women to this pesticide are also available.

In this study the possibility of lipid peroxidation in the human placental microsomes, the biochemical 
mechanism proposed for the pulmonary toxicity of paraquat (Bus and Gibson, 1979), was examined in vitro. The results indicated that paraquat can induce deleterious lipid peroxidation process in the human placental microsome. The process was proteindependent and required NADPH and reduced transitional metal. The absence of initial lag in the accumulation of measurable MDA suggests that placental microsomes are uniquely susceptible to the radical attacks due probably to inadequate protection by the endogenous antioxidants. The biological significance of these findings remains to be established.

Acknowledgements - This work was supported in part by the Rackham Faculty Research Grant and by the Grant T32 ES 07062 from the U.S. Public Health Service, National Institute of Health.

\section{REFERENCES}

Aust S. D., Morehouse, L. A. and Thomas, C. E. (1985) Role of metals in oxygen radical reactions. J. Free Rad. biol. Med. 1, 3-25.

Awasthi Y. C., Dao D. D., Lal A. K. and Srivastava S. K. (1979) Purification and properties of glutathione peroxidase from human placenta. Biochem. J. 177, 471-476.

Benedetti A., Comporti M., Fulceri R. and Esterbauer H. (1984) Cytotoxic aldehydes originating from the lipid peroxidation of liver microsomal lipids. Identification of 4,5-dihydroxydecenal. Biochim. biophys. Acta 792, $172-181$.

Braughler J. M., Duncan L. A. and Chase R. L. (1986) The involvement of iron in lipid peroxidation. Importance of ferric to ferrous ratios in initiation. J. biol. Chem. 261, 10282-10289.

Bus J. S., Aust S. D. and Gibson J. E. (1974) Superoxideand singlet oxygen-catalyzed lipid peroxidation as a possible mechanism for paraquat toxicity. Biochem. biophys. Res. Commun. 58, 749-755.

Bus J. S., Preache M. M., Cagen S. Z., Posner H. S., Eliason B. C., Sharp C. W. and Gibson J. E. (1975) Fetal toxicity and distribution of paraquat and diquat in mice and rats. Toxic. appl. Pharmac. 33, 450-460.

Bus J. S. and Gibson J. E. (1979) Lipid peroxidation and its role in toxicology. Rev. Biochem. Toxic. 1, 125-149.

Diamant S., Kissilevitz R. and Diamant Y. (1980) Lipid peroxidation system in human placental tissue: General properties and the influence of gestational age. Biol. Reprod. 23, 776-781.

Finnelly J. J., Gallagher J. T. and Carroll R. J. (1968) Paraquat poisoning in a pregnant women. Br. Med.J. 3, 722-723.

Gornall A. G., Bardawill C. J. and David M. M. (1949) Determination of serum proteins by means of the Biuret reaction. J. biol. Chem. 177, 751-760.

Haley T. J. (1979) Review of the toxicology of paraquat ( $l l^{\prime}$-dimethyl-4,4'-bipyridinium chloride). Clin. Toxic. 14, $1-46$.

Hoffmann D. J. and Eastin W. C. (1982) Effects of lindane, paraquat, toxaphene, and 2,4,5, trichlorophenoxyacetic acid on mallard embryo development. Archs Envir. Contam. Toxic. 11, 79-86.

Ilett K. F., Stripp G., Menard R. H., Reid W. D. and Gillette J. R. (1974) Studies on the mechanism of the lung toxicity of paraquat. Comparison of tissue distribution and some biochemical parameters in rats and rabbits. Toxic. appl. Pharmac. 28, 216-226.

Ingebrigtsen K., Nafstad I. and Andersen R. A. (1985) Distribution and transplacental transfer of paraquat in rats and guinea-pigs. Gen. Pharmac. 15, 201-204.
Kenel M. F. and Kulkarni A. P. (1982) Effects of paraquat on the NADPH-dependent lipid peroxidation in human placental microsomes. Joint ASPET/SOT Meeting Louiseville, Kentucky, Abst. No. 429.

Klimek J., Schaap A. P. and Kimura T. (1982) Inhibition of lipid peroxidation by paraquat: site of inhibition in the cytochrome P-450-dependent steroid hydroxylase system from bovine adrenal cortex mitochondria. Biochem. biophys. Res. Commun. 107, 499-505.

Kornbrust D. J. and Mavis R. D. (1980) The effect of paraquat on microsomal lipid peroxidation in vitro and in vivo. Toxic. appl. Pharmac. 53, 323-332.

Kulkarni A. P. and Hodgson E. (1981) A comparison of NADPH and cumene hydroperoxide-stimulated lipid peroxidation in mouse hepatic microsomes. Int. J. Biochem. 13, 811-816

Kulkarni A. P., Strohm B. and Houser W. H. (1985) Human placental indanol dehydrogenase: Some properties of the microsomal enzyme. Xenobiotica 15, 513-519.

Kulkarni A. P. and Kenel M. F. (1987) Human placental lipid peroxidation. I. Some characteristics of the NADPH-supported microsomal reaction. Gen. Pharmac. In press.

Matkovics B., Fodor I., and Kovacs K. (1975) Properties of enzymes. III. A comparative study of peroxide decomposing enzymes and superoxide dismutase of human placenta at different ages. Enzyme 19, 285-293.

Misra S. and Mukherjea M. (1985) A comparative study of human placental and fetal liver catalase during development. Eur. J. Obstet. Gynecol. Reprod. Biol. 19, 81-88.

Misra S. and Mukherjea M. (1985) A comparative study of human placental and fetal liver catalase during development. Eur. J. Obstet. Gynecol. Reprod. Biol. 19, 81-88.

Montgomery M. R. and Niewoehner D. E. (1979) Oxidantinduced alterations in pulmonary microsomal mixed function oxidation: Acute effects of paraquat and ozone. J. Environ. Hlth Sci. C13, 205-219.

Moody C. S. and Hassan H. M. (1982) Mutagenicity of oxygen free radicals. Proc. natn. Acad. Sci. U.S.A. 79, 2855-2859.

Musson F. A. and Porter C. A. (1982) Effect of ingestion of paraquat on a 20 -week gestation fetus. Postgrad. Med. J. 58, 731-732.

Nelson J. L. and Kulkarni A. P. (1986) Partial purification of human placental peroxidase by affinity chromatog raphy. Toxicologist 6, 144 .

Plaa G. L. and Witschi H. (1976) Chemicals, drugs and lipid peroxidation. A. Rev. Pharmac. 16, 125-141.

Radulovic L. L. and Kulkarni A. P. (1985) A rapid, novel high performance liquid chromatography method for the purification of glutathione $S$-transferase: An application to the human placental enzyme. Biochem. biophys. Res. Commun. 128, 75-81.

Radulovic L. L. and Kulkarni A. P. (1986) High performance liquid chromatographic separation and study of the charge isomers of human placental glutathione $S$ transferase. Biochem. J. 239, 53-57.

Sekiba K. and Yoshioka T. (1979) Changes of lipid peroxidation and superoxide dismutase activity in the human placenta. Am. J. Obstet. Gynecol. 135, 368-371.

Selypes A., Nagymajtenyi B. and Berencsi G. (1982) Mutagenic and embryotoxic effects of paraquat and diquat. Bull. Envir. Contam. Toxic. 25, 513-517.

Shamberger R. J., Andeone T. L. and Willis C. E. (1974) Antioxidants and cancer. IV. Initiating activity of malonaldehyde as a carcinogen. J. Natn. Cancer Inst. 53, 1771-1773.

Shu H., Talcott R. E., Rice S. A. and Wei E. T. (1979) Lipid peroxidation and paraquat toxicity. Biochem. Pharmac. 28, 327-331.

Slater T. F. (1984) Free-radical mechanisms in tissue injury. Biochem. J. 222, 1-15. 
Smith P. and Heath D. (1976) Paraquat. CRC Rev. Toxic. 4, 411-445.

Steffen C. and Netter K. J. (1980) On the mechanism of paraquat action on microsomal oxygen reduction and its relation to lipid peroxidation. Toxic. appl. Pharmac. 53, 323-332.

Talcott R. E., Shu H. and Wei E. T. (1979) Dissociation of microsomal oxygen reduction and lipid peroxidation with the electron acceptors, paraquat and menadione. Biochem. Pharmac. 28, 665-671.

Trush M. A., Mimnaugh E. G. Ginsburg E. and Gram T. E. (1981) In vitro stimulation by paraquat of reactive oxygen-mediated lipid peroxidation in rat lung microsomes. Toxic. appl. Pharmac. 60, 279-286. 